http://jmscr.igmpublication.org/home/

ISSN (e)-2347-176x ISSN (p) 2455-0450

crossref DOI: https://dx.doi.org/10.18535/jmscr/v9i5.36

Journal Of Medical Science And Clinical Research

\title{
Progressive Familial Intrahepatic Cholestasis Presenting With Intra Cranial Bleed
}

\author{
Authors \\ Dr Ankita Kamble [Resident MD Pediatrics]*, Dr Shaista Parween [Assistant Prof, \\ GGMC \& Sir JJH, MD Pediatrics], Dr D. Ragadeepika, Dr Harpreet Kaur, \\ Dr Ashutosh Pai, Dr Vinita Tripathi \\ *Corresponding Author \\ Dr Ankita Kamble
}

\begin{abstract}
Progressive familial intrahepatic cholestasis [PFIC] refers to heterogeneous group of autosomal recessive disorders of childhood that disrupts bile formation and present with cholestasis of hepatocellular origin. The exact prevalence is unknown, but the estimated incidence varies from between 1/50,000 and 1/100,000. The following case highlights the importance of combined clinical, pathological and genetic approach to the diagnosis of PFIC. We report this 5-month-old male child, born of nonconsanguineous marriage presented with lethargy, unable to take feeds and 1 episode of projectile vomiting since past 12 hours. On examination child was drowsy with paucity of movements of left upper and lowerlimb, right eye ptosis, child was pale and yellowish discoloration of body, liver span was around $9 \mathrm{~cm}$. CT Scan was done suggestive of s/o right frontal -temporoparietal intracranial haemorrhage. Detailed investigations were done showed anaemia with direct hyperbilirubinemia with deranged PT-INR which was corrected after giving vitamin K. Rest of the liver parameters including GGT was normal. Mother was giving strong history of previous child death at the age of 11 months due to some liver disease. PFIC was considered as one of the differentials. Liver biopsy showed ballooning degeneration and cholestasis, bile plugging in bile canaliculi seen, mild portal fibrosis seen with dilatation of central vein noticed s/o PFIC.Genetic work up was sent which confirmed diagnosis of PFIC type -2.
\end{abstract}

Keywords: PFIC, gene, pruritus, cholestasis, jaundice.

\section{Introduction}

Progressive familial intrahepatic cholestasis (PFIC) or Byler disease is an autosomal recessive inherited condition, characterized by inability to drain bile from the liver even though the large bile ducts are open (i.e., cholestasis). It is a very rare cause of chronic liver disease in children and usually begins in infants less than 6 months of age. However, some may present in later childhood. We present a case of PFIC type -2 who was presented with intra -cranial hemorrhage and was managed succefully.

\section{Case Report}

5 Months old male child $\mathrm{xxx}, 3^{\text {rd }}$ birth by order, born of non-consanguineous marriage was brought by parents in casualty with complains of yellowish discoloration since 1 month, feeding 
difficulty and lethargy since 1 day. 1 episode of projectile vomiting 1 day back, child had no history of admissions in the past, no h/o blood transfusions. Child had yellow coloured stools and high coloured urine .Mother was giving history of previous female child death due to some liver disease, papers not available with parents .Birth history was normal without any postnatal complications. Child was immunised up to 6 weeks (incompletely immunised) and child was exclusively breastfed by mother. Child had a normal developmental history. On physical examination child was lethargic, pale, yellowish tinge, with right eye ptosis, reduced movement of left upper and lower limb. The pulse rate 160/min, peripheral pulses were well felt, respiratory rate- $34 / \mathrm{min}$, blood pressure was 70/40 mmhg in right upper arm in supine position. Weight was $5.6 \mathrm{~kg}$ and height $-59 \mathrm{~cm}, \mathrm{Z}$-score was between -2 to -3 SD. Child had no signs of fat-soluble vitamin deficiency, no scratch marks seen, no clubbing, no cyanosis seen. Liver was enlarged with a span of $9 \mathrm{~cm}$ in midclavicular line, non-tender, firm in consistency. The spleen was not palpable, no ascites present. The infant was drowsy, brisk deep tendon reflexes in lower limbs. There were no signs of meningeal irritation.

Laboratory evaluation was done showed following values: Haemoglobin - $8.7 \mathrm{~g} / \mathrm{dl}$, WBC$30,000 / \mathrm{cmm}$, Platelet count - 5.2 lakhs, serum electrolytes - sodium-132, potassium - 3.7, liver function test - SGOT - 146, SGPT - 52, Bilirubintotal-6.6, direct -3.4, PT -16.9, INR - 1.37, Albumin-3.3, GGT -24.6, RFT -WNL,RBS $106 \mathrm{mg} / \mathrm{dl}$, S.Ammonia - 70.Child was kept nil by mouth, was started on IV fluids with dextrose, started on antibiotics - Inj Taxim, inj amikacin, O2- by nasal prongs . Urgent CT -brain was done - s/o right frontal -temporoparietal intracranial haemorrhage of size $4.4 \times 3.3 \times 3.3 \mathrm{~cm}$ leading to midline shift of $11 \mathrm{~mm}$.Inj vitamin $\mathrm{K}$ was given due to deranged coagulation profile .Pre-operative work up was done, blood was arranged, blood for coagulation profile was taken, 1 unit of PCV was transfused, Urgent cerebral angiogram was done to rule out tumour apoplexy and to look for any bleed in dural venous sinuses. Craniotomy and lax duraplasty was done under GA. Post op repeat Ctscan was done s/o midline shift of $6 \mathrm{~mm}$, with rest of findings consistent with previous CT. Child was kept NBM/IVM, O2 nasal prongs, antibiotics continued, child had lip smacking movements post op, hence child was loaded with inj. levipil and f/b maintenance dose, child had electrolyte imbalance - low sodium, hence started on 3\% Nacl with serum electrolyte monitoring twice daily, once stabilised it was omitted. During course of treatment child developed, oedema over b/l lower and upper limb, scrotal oedema present, repeat LFT was done s/o increased enzymes, reduced albumin levels to $1.6 \mathrm{gm} \%$, hence child was transfused with albumin, During course of treatment oedema reduced, right eye ptosis resolved, movements in left upper and lower limb improved. Child was started on OGT feeds initially, later on was allowed orally, weaning was started, cholestatic regimen [ADEK] was added. During course of treatment irritability of child was increased with inconsolable cry, hence repeat ammonia was sent -155 , hence child was kept NBM / IVM, started on lactulose, sodium benzoate, child responded, repeat ammonia within normal limits, child started on oral feeds lactulose and sodium benzoate omitted. During stay in hospital, it was noticed that child has pruritus, hence started on urodeoxycholic acid, did not respond to it, hence was started on cholestyramine to which child has responded. Usg abdomen s/o - moderate hepatomegaly. TORCH titre of both mother and child was sent came out to be negative Once child stabilised, child was planned for liver biopsy.

Liver biopsy was s/omost of the hepatocytes shows ballooning degeneration and cholestasis, bile plugging in bile canaliculi seen, mild portal fibrosis seen with dilatation of central vein noticed s/o PFIC. Child has no history of loose stools, GGT is WNL, BERA was WNL, thyroid profile WNL. Pediatric gastroenterology opinion was taken, advised to sent genetic work up (WHOLE 
EXOME SEQUENCING), counselling for liver transplant was done. Genetic w/u was sent, report awaited. The above findings, along with the clinical and laboratory data were s/o PFIC TYPE 2.

Child was discharged on UDCA, cholestyramine, Vitamins -ADEK, skin moisturizing lotions. Parents were counselled on the need for liver transplantation and importance of antenatal testing in future pregnancy.

\section{Discussion}

The common differential diagnosis for such presentations are extrahepatic biliary obstruction, metabolic disorders, infective pathology. TORCH infections were ruled out by doing TORCH titre in child and mother. Ultrasonography ruled out extra-hepatic cause of cholestasis. Bleeding $d / o$ were ruled out by doing coagulation profile and haemophilia profile .Intra -cranial haemorrhage was probably due to deranged coagulation profile which was corrected after giving vitamin $\mathrm{K}$. Thyroid d/o was ruled out .Due to strong family history of previous sibling death due to some liver d/o, we had planned to r/o metabolic d/o by sending TMS (Tandem Mass Spectroscopy) and urinary GCMS (Gas chromatography - mass spectroscopy) and had done liver biopsy, liver biopsy was s/o most of the hepatocytes shows ballooning degeneration and cholestasis, bile plugging in bile canaliculi seen, mild portal fibrosis seen with dilatation of central vein noticed s/o PFIC.

In addition to PFIC, there are other causes of intra hepatic cholestasis without morphological changes of intra-hepatic ducts: hypothyroidism, peroxisomal d/o, Niemann -pick disease, alpha 1 antitrypsin deficiency, cystic fibrosis, galactosemia and tyrosinemia. The clinical and laboratory findings including liver biopsy were not in consistent with these disorders. Thus, clinical presentation, GGT - WNL, strong family history, histopathological examination of liver biopsy was s/o PFIC type -2 and Genetic report
(WHOLE EXOME SEQUENCING) confirmed the diagnosis of PFIC type -2 .

PFIC is caused by defects in several genes that produce proteins needed for bile formation and transportation or flow of bile throughout the body. A defect leads to blockage of bile in liver or bile ducts, damaging liver cells and causing a dangerous build up of waste in the blood stream. Decreased bile flow also prevents the body from being able to properly absorb fats and vitamins. Common symptoms of PFIC include, severe itching caused by buildup of bile salts in body, disproportionate jaundice, and poor weight gain [due to lack of bile needed to digest and absorb fat] and poor growth.

The defect is in ATP8B1 gene encoding the FIC 1 protein, $\mathrm{ABCB} 11$ gene encoding $\mathrm{BSEP}$ protein and $\mathrm{ABCB} 4$ gene encoding MDR3 protein in PFIC 1,2 and 3 respectively. The basic defect is impaired bile salt secretion in PFIC1/2 whereas in PFIC3, it is reduced biliary phospholipid secretion. The main clinical presentation is in the form of cholestatic jaundice and pruritus. serum gamma glutamyl transpeptidase [GGT] is normal in PFIC 1 and 2, while it is raised in PFIC3.The incidence of PFIC presenting with intra cranial bleed is less, but cases are seen due to secondary deficiency of vitamin $\mathrm{K}$.

Treatment includes nutritional support (adequate calories, supplementation of at soluble vitamins and medium chain triglycerides) and use of medications to relieve pruritus as initial therapy followed by biliary diversion procedures in selected patients. Ultimately liver transplantation is needed in most of the patients as they develop progressive liver fibrosis, cirrhosis and end stage liver disease. Due to the high risk of developing liver tumors in PFIC2 patients, monitoring recommended from infancy. Mutation targeted pharmacotherapy, gene therapy and hepatocyte transplantation are being explored as future therapeutic options. 


\section{Acknowledgment}

We would like to thank Dr. Bela Verma, Head of Department of pediatrics to allow us publish this manuscript.

\section{Reference}

1. Whitington PF, Freese DK, Alonso EM, Schwarzenberg SJ, SharpHL. Clinical and and biochemical findings in progressive familial intrahepatic cholestasis. J Pedia tr Gastroentrol Nutr 1994;18:134-41.

2. Balis treri WF. Inborn errors of bile acid biosynthesis and transport. Novel forms of metabolic liver.J Gastroenterol Hepatol 1999; 14:594-9

3. Davit-SprauL A, GonzalesE, BaussanC, JacqueminProgressivefamilial intra hepatic cholestasis. Orphanet J Rare Dis 2009; 4:1.

4. Emerick KM, Whitington PF. Molecular basis of neonatal cholestasis. Pediatr Clin North Am 2002; 14:594-9.

5. Jacquemin E. Progressive familial intra hepatic cholestasis. J Gastroenterol Hepatol 1999; 14:594-9

6. Dollance T,Akyol G, Bulac S, Progressive intra hepatic cholestasis with normal GGT level appearing with lichenification and enlargement of hands and feet's .Turk J Pediatr2005:47:385-9.

7. Ismail $\mathrm{H}$, Kalicipski $\mathrm{P}$, Markiewicz, jankowska 1,Pawowska J, Kluge P,et al. Treatment of progressive intrahepatic cholestasis. 